\title{
On the Gauss Map of Complete Minimal Surfaces with Finite Total Curvature
}

\author{
YI FANG
}

\begin{abstract}
We prove that if $M$ is an immersed complete minimal surface in $\mathbb{R}^{3}$, and the total curvature of $M$ is larger or equal to $-20 \pi$, then the Gauss map of $M$ can only miss at most two values in the Riemann sphere $\Sigma=\mathbb{C} \cup\{\infty\}$. In general, we prove that if all the ends of $M$ are embedded, and the Gauss map of $M$ misses three values, then the number of flat ends of $M$ is larger than 2. Finally, we give an example of a properly immersed singly periodic minimal surface whose Gauss map misses three values.
\end{abstract}

1. Introduction. The study of the global properties of complete, immersed minimal surfaces in $\mathbb{R}^{3}$, especially the properties of their Gauss map, has achieved many important results and also given rise to many problems in recent decades. Among them a very interesting problem is the following: if $X: M \rightarrow \mathbb{R}^{3}$ is a complete non-planar minimal immersion, and $N: M \rightarrow S^{2} \cong \mathbb{C} \cup\{\infty\}=\Sigma$ its Gauss map, how many values can $N$ miss?

In 1961 Osserman [13] proved that $N$ can miss at most a set of points of logarithmic capacity zero. In 1981 Xavier [19] proved that $N$ can miss at most 6 values. In 1988 Fujimoto [6] proved that $N$ can miss at most 4 values. This is the best possible number, since there are a lot of examples of complete minimal immersions whose Gauss map misses 4 values. An example is Scherk's surface, which is even embedded. See [15] (page 72, Theorem 8.3) for other examples. However, all of these examples have infinite total Gauss curvature. This is not a surprise, since in 1964 Osserman [14] proved that if $M$ has finite total curvature, then $N$ can miss at most 3 values.

The catenoid has finite total curvature of $-4 \pi$ and its Gauss map misses 2 values. There is no known example of a complete immersed minimal surface of finite total curvature whose Gauss map misses 3 values. Hence either 2 or 3 is the maximal number of values that may be omitted. Since Osserman posed this problem in 1967, many years have passed without any progress. To look 
for an answer to this question, one naturally seeks necessary conditions for the existence of such a surface. However, few advances in this direction have been made during the last two decades. Then in 1987, Weitsman and Xavier [18] proved that if $N$ misses 3 values, the total curvature of $M$ is less than or equal to $-16 \pi$. In this paper we shall prove that, in fact, the total curvature of $M$ is less than or equal to $-20 \pi$.

A result of Osserman [13] says that if $M$ is a complete, oriented, minimal surface in $\mathbb{R}^{3}$, then the total Gauss curvature is an integer multiple of $4 \pi$. For a nonorientable minimal surface $M$, there is no well defined Gauss map to $S^{2}$ on $M$. However, its oriented double covering, $\tilde{M}$, is a complete, orientable minimal surface. We can study the image of the Gauss map of $\tilde{M}$. Hence we only consider the orientable case.

Let $X: M \rightarrow \mathbb{R}^{3}$ be a complete minimal immersion with finite total curvature. Osserman [13] proved that $M$ is conformally diffeomorphic to a genus $k$, compact Riemann surface $S_{k}$ with finite number of punctures, i.e., $M \cong S_{k}-\left\{p_{1}, \ldots, p_{r}\right\}$. Let $D_{i}, i=1, \ldots, r$, be small disk-neighborhoods of $p_{i}$. We will call each $E_{i}=X\left(D_{i}-\left\{p_{i}\right\}\right)$ an end of $M$. If $X$ is an embedding on $D_{i}-\left\{p_{i}\right\}$, then $E_{i}$ approaches asymptotically a catenoid or a plane, [17], we call such an end a catenoid end or flat end, respectively. We shall also prove that if the Gauss map of $M$ misses 3 values and $M$ has $r$ embedded ends, $\ell$ of which are catenoid ends, then $r-\ell>2$.

In the following we need the knowledge of the Enneper-Weierstrass representation. For the details of this theory, we refer to either [12] or [15]. Let $M$ be a Riemann surface, $g$ be a meromorphic function on $M$, and let $\eta$ be a holomorphic 1-form on $M$, such that whenever $g$ has a pole at $p \in M$ of order $m, \eta$ has a zero at $p$ of order $2 m$, and $\eta$ has no other zeros. Define holomorphic 1-forms on $M$ as follows,

$$
\omega_{1}=\frac{1}{2}\left(1-g^{2}\right) \eta, \quad \omega_{2}=\frac{i}{2}\left(1+g^{2}\right) \eta, \quad \omega_{3}=g \eta .
$$

Then if for any loop $\gamma$ on $M$,

$$
\operatorname{Re} \int_{\gamma} \omega_{i}=0, \quad i=1,2,3,
$$

the following map gives an minimal immersion of $M$ in $\mathbb{R}^{3}$,

$$
X(p)=\operatorname{Re} \int_{p_{0}}^{p}\left(\omega_{1}, \omega_{2}, \omega_{3}\right)
$$

where $p_{0} \in M$ is a fixed point. On the other hand, any minimal immersion can be represented this way, and the function $g$ in (1.1) is the Gauss map of the immersion. For the details, please see [15]. Moreover, we have the following classical proposition: 
Proposition 1.1. Let $S_{k}$ be a genus-k Riemann surface,

$$
M=S_{k}-\left\{p_{1}, \ldots, p_{r}\right\}
$$

and

$$
X: M \rightarrow \mathbb{R}^{3}
$$

be a complete minimal immersion of finite total curvature, where $X$ is defined as in (1.2). Then the Gauss map $g$ can be extended to $S_{k}$ as a meromorphic function. Suppose that $E_{i}$ is an embedded end corresponding to $p_{i}$. If $g$ has a pole of order $m \geq 1$ at $p_{i}$, then $\eta$ has a zero of order $2 m-2$ at $p_{i}$. If $g$ takes on a finite value at $p_{i}$, then $\eta$ has a pole of order 2 at $p_{i}$. Furthermore, $p_{i}$ is a branch point of $g$, if and only if $E_{i}$ is a flat end.

Moreover, the Gauss curvature of $M$ is given as

$$
K(p)=-\left[\frac{4\left|g^{\prime}(p)\right|}{|f(p)|\left(1+|g(p)|^{2}\right)^{2}}\right]^{2}
$$

where $\eta=f(z) d z$ in a local coordinate neighborhood.

For the proof of this proposition, please see [3] or [4], and for example, see [15].

2. General Results. Let $X: M \rightarrow \mathbb{R}^{3}$ be a complete immersed minimal surface in $\mathbb{R}^{3}$ of finite total curvature. By a theorem of Osserman [13],

$$
M=S_{k}-\left\{p_{1}, \ldots, p_{r}\right\}
$$

where $S_{k}$ is a compact Riemann surface of genus $k$. Let

$$
g: M \rightarrow \Sigma \cong S^{2}
$$

be the Gauss map of $M$. Then $g$ can be extended to $S_{k}$ and is a meromorphic function. Assume $g$ misses $\ell$ values, $q_{1}, \ldots, q_{\ell} \in \Sigma$, on $M$.

Since $g$ is surjective on $S_{k}$, it must be

$$
\left\{q_{1}, \ldots, q_{\ell}\right\} \subset g\left(\left\{p_{1}, \ldots, p_{r}\right\}\right)
$$

and $r \geq \ell$. Let $\operatorname{deg} g=n$. By [8],

$$
C(M)=-4 n \pi=-2 \pi\left(r+\sum_{i=1}^{r} I_{i}-2(1-k)\right)
$$


where $C(M)$ is the total Gauss curvature of $M$ and $I_{i}$ is the multiplicity of the end $E_{i}$ corresponding to $p_{i}, i=1, \ldots, r, I_{i} \geq 1, I_{i}=1$ if and only if $E_{i}$ is embedded. Using (2.4), we get

$$
2 n=r+\sum_{i=1}^{r} I_{i}-2(1-k)
$$

The total branching order of $g$ is, by the Hurwitz formula,

$$
n_{0}=2(n+k-1)
$$

Let $n_{1}$ be the total branching order among $\left\{p_{1}, \ldots, p_{r}\right\}$. Then $n_{1} \leq n_{0}$. Since $\operatorname{deg} g=n$, we need $\ell \times n$ points (counting multiplicity) to cover $\left\{q_{1}, \ldots, q_{\ell}\right\}$. Since

$$
\left\{p_{1}, \ldots, p_{r}\right\} \subset g^{-1}\left(\left\{q_{1}, \ldots, q_{\ell}\right\}\right)
$$

we have

$$
r \geq \ell n-n_{1} \geq \ell n-n_{0}=(\ell-2) n+2(1-k)
$$

that is,

$$
r+2(k-1) \geq(\ell-2) n
$$

By (2.5),

$$
2 n-\sum_{1}^{r} I_{i}=r+2(k-1) \geq(\ell-2) n
$$

i.e.,

$$
(\ell-4) n \leq-\sum_{1}^{r} I_{i} \leq-r<0 .
$$

Since $n \geq 0$, we get $\ell<4$. Summarizing, we have the following proposition:

Proposition. Suppose $X: M \rightarrow \mathbb{R}^{3}$ is a complete, immersed minimal surface with total curvature $C(M)=-4 n \pi$. Let $g: M \rightarrow \Sigma$ be the Gauss map. Then $M=S_{k}-\left\{p_{1}, \ldots, p_{r}\right\}$, where $k$ is the genus of $S_{k} . g$ can miss at most three values of $\Sigma$. If $g$ misses three values of $\Sigma$, then

(1) (Osserman [14]) $k \geq 1$ and $n \geq r \geq 3$.

(2) If $k=1$, then $n=r$, and $I_{i}=1, i=1, \ldots, r$; that is, each end of $M$ is an embedded end.

(3) (Osserman [14]) The total curvature of $M$ satisfies $C(M) \leq-12 \pi$. 
Proof. Because $\ell<4, g$ can miss at most three values of $\Sigma$. Now, supposing that $g$ misses three values, $(2.8)$ gives

$$
n \geq \sum_{i=1}^{r} I_{i} \geq r .
$$

If $k=0$, then by (2.7) and (2.9) we have both $r \geq n+2$ and $n \geq r$, a contradiction.

If $k=1,(2.7)$ and (2.9) force $r=n$. By (2.9) we also have $I_{i}=1$. From [8] we know that this implies that each end of $M$ is an embedded end. Noticing that $r \geq 3$ and $k \geq 1$, we have by (2.4),

$$
C(M) \leq 4 \pi(1-k-r) \leq-12 \pi \text {. }
$$

Proposition $2.2([7])$. Assume $X: M \rightarrow \mathbb{R}^{3}$ is a complete immersed minimal surface in $\mathbb{R}^{3}$ of finite total curvature $C(M)=-4 n \pi$ and $M=S_{k}$ $\left\{p_{1}, \ldots, p_{r}\right\}$. If the Gauss map $g$ of $M$ misses 3 values and $k=1$, then for any point $p \in M, K(p)<0$, i.e., $g: M \rightarrow S^{2}-\left\{q_{1}, q_{2}, q_{3}\right\}$ is a regular covering. Moreover,

$$
\left\{p_{1}, \ldots, p_{r}\right\}=g^{-1}\left(\left\{q_{1}, q_{2}, q_{3}\right\}\right)
$$

Proof. Since for a minimal surface $K(p) \leq 0$, we only need to prove $K(p)$ is never zero. By (1.3), we have

$$
K(p)=-\left[\frac{4\left|g^{\prime}(p)\right|}{|f(p)|\left(1+|g(p)|^{2}\right)^{2}}\right]^{2},
$$

where $f(z) d z=\eta$ is the local data in the Enneper-Weierstrass representation formula. Thus $K(p)=0$ if and only if $p$ is a branch point of $g$. Therefore, we only need to prove that $g$ has no branch points on $M$. By (2.6) and Proposition 2.1, $r=n$, and

$$
n_{0}=2(n+k-1)=2 n=2 r,
$$

where $n_{0}$ is the total branching order of $g$.

Suppose $g$ misses $q_{1}, q_{2}, q_{3} \in \Sigma$ on $M$. Then

$$
\left\{q_{1}, q_{2}, q_{3}\right\} \subset g\left(\left\{p_{1}, \ldots, p_{r}\right\}\right) .
$$

Because $r=n=\operatorname{deg} g$, in order to cover $\left\{q_{1}, q_{2}, q_{3}\right\} r$ times, we need $3 r$ points (counting multiplicities). But, by assumption,

$$
g^{-1}\left(\left\{q_{1}, q_{2}, q_{3}\right\}\right) \subset\left\{p_{1}, \ldots, p_{r}\right\}
$$

So the total branching order $n_{1}$ among $\left\{p_{1}, \ldots, p_{r}\right\}$ satisfies $r+n_{1}=3 r$; i.e., $n_{1}=2 r=n_{0}$, and $\left\{p_{1}, \ldots, p_{r}\right\}=g^{-1}\left(\left\{q_{1}, q_{2}, q_{3}\right\}\right)$. Thus if $p \in M$, then $p$ is not a branch point. This completes the proof of the proposition.

Now we can prove our first main theorem. 
Theorem 2.1. Let $X: M \rightarrow \mathbb{R}^{3}$ be a complete, immersed minimal surface in $\mathbb{R}^{3}$ of finite total curvature with $r$ embedded ends, $\ell$ of which are catenoid ends. If the Gauss map $g$ of $M$ misses three values, then $r-\ell>2$.

Proof. Let $M=S_{k}-\left\{p_{1}, \ldots, p_{r}\right\}, E_{i}$ be the end corresponding to $p_{i}$. We know that if $E_{i}$ is a catenoid end, then $g$ has no branch at $p_{i}$. If $r-\ell \leq 2$, then at most two of the $\left\{p_{1}, \ldots, p_{r}\right\}$ are branch points of $g$. Hence at least one of $\left\{q_{1}, q_{2}, q_{3}\right\}$ is a regular value of $g$, say $q_{1}$. There should be $n$ different points $\left\{P_{1}, \ldots, P_{n}\right\}$ of $S_{k}$ which are mapped to $q_{1}$ by $g$. But $g$ misses $q_{1}$ on $M$. Hence these points must be contained in $\left\{p_{1}, \ldots, p_{r}\right\}$. In particular, $n \leq r$. By Proposition 2.1, $n \geq r$, thus $n=r$ and $\left\{P_{1}, \ldots, P_{n}\right\}=\left\{p_{1}, \ldots, p_{r}\right\}$. But this is to say that $g$ misses $q_{2}$ and $q_{3}$ on $S_{k}$, which is impossible. This contradiction proves the theorem.

Theorem 2.2. Let $X: M \rightarrow \mathbb{R}^{3}$ be a complete, immersed minimal surface of finite total curvature with $r$ ends, all of them embedded. $M=S_{k}-\left\{p_{1}, \ldots, p_{r}\right\}$. Let $N: S_{k} \rightarrow S^{2}$ be the extended Gauss map. Let $E_{i}$ be the corresponding end at $p_{i}$, and $N_{i}=N\left(p_{i}\right)$. Suppose that $E_{j}, j=1,2, \ldots, \ell$, are catenoid ends, $E_{i}, i=\ell+1, \ldots, r$, are flat ends. Then there exist nonzero real numbers $a_{j}$, $j=1,2, \ldots, \ell$, such that

$$
\sum_{j=1}^{\ell} a_{j} N_{j}=\overrightarrow{0}
$$

Remark 2.1. This result was known to Schoen and Kusner in 1982, and is contained both in [9] and [10] as special cases of constant mean curvature balancing. Since the proof of this theorem is just a modified version of the proof in [16], in which all the ends are assumed catenoid ends, we omit the proof here. The readers can see [4] and [16] for the details.

Corollary 2.1 (Also see [10]). There is no complete, immersed minimal surface in $\mathbf{R}^{3}$, with finite total curvature and embedded ends that has just one catenoid end, no matter how many flat ends it has.

Proof. If not, we have $a N=\overrightarrow{0}$ and $a \neq 0$, which is impossible.

Theorem 2.1 and Corollary 2.1 have a useful corollary.

Corollary 2.2. Let $X: M \rightarrow \mathbb{R}^{3}$ be a complete immersed minimal surface in $\mathbb{R}^{3}$ of finite total curvature with at most 4 ends, all of them are embedded. If the Gauss map of $X$ misses three values, then all ends must be flat

Proof. By Theorem 2.1, $M$ can have at most one catenoid end. But by Corollary 2.1, $M$ cannot have just one catenoid end. 
3. The Case of $C(M)=-16 \pi$. In [18] Weitsman and Xavier proved that if $M$ is a complete, immersed minimal surface of finite total curvature, whose Gauss map misses three values, then the total Gauss curvature of $M$ satisfies $C(M) \leq-16 \pi$. In this section we will prove that their result can be improved to $C(M) \leq-20 \pi$, that is, our second main theorem:

Theorem 3.1. Let $M$ be a complete minimal surface of finite total curvature. If the Gauss map of $M$ misses 3 values, then

$$
C(M) \leq-20 \pi
$$

Proof. Since $C(M)=-4 n \pi, n \in \mathbb{Z}^{+}$, by the result of Weitsman and Xavier, we need only to consider the case that $C(M)=-16 \pi$. The following lemma and next two subsections will prove this theorem.

Lemma. Suppose that $M$ is a complete, minimal immersed surface with $C(M)=-4 n \pi$ and whose Gauss map misses three values. Suppose $M=S_{k}-$ $\left\{p_{1}, \ldots, p_{r}\right\}$, where $S_{k}$ is a genus- $k$ closed Riemannian surface, then $k \leq n-2=$ $-(C(M) / 4 \pi)-2$. If $C(M)=-16 \pi$, then either

$$
M=T-\left\{p_{0}, p_{1}, p_{2}, p_{3}\right\}
$$

where $T$ is a torus, or

$$
M=S_{2}-\left\{p_{1}, p_{2}, p_{3}\right\}
$$

In both cases, the ends of $M$ are embedded flat ends.

Proof. By (2.5), $r+\sum_{i=1}^{r} I_{i}+2(k-1)=2 n$. Since $r \geq 3$ and $I_{i} \geq 1$, it follows that $r+\sum_{i=1}^{r} I_{i} \geq 6$, and $2 n \geq 6+2(k-1)$. Hence $k \leq n-2$.

Now suppose $n=4$, then $k \leq 2$. By Proposition 2.1, $k \neq 0$. If $k=1$, by Proposition 2.1, $r=n=4$ and each end is embedded. If $k=2$, then $2(k-1)=2$, thus $r+\sum_{1}^{r} I_{i}=2 n-2(k-1)=6$. The only possibility is $r=3, I_{i}=1$, for $i=1,2,3$. Which means that each end is embedded. By Corollary 2.2 , in both cases the ends are flat ends. The proof of the lemma is completed.

3.1. The Genus-1 Case. We will need to use the properties of the Weierstrass $\wp$ function and the related Zeta function, for which we refer to [1] and [11].

Assume $M=T-\left\{p_{0}, p_{1}, p_{2}, p_{3}\right\}$ is a torus $T$ with 4 punctures. Let $X$ : $M \rightarrow \mathbb{R}^{3}$ be a complete, minimal immersion with finite total curvature. Let $g: M \rightarrow \mathbb{C} \cup\{\infty\}$ be the Gauss map. Assume $g$ misses 3 values $q_{1}, q_{2}, q_{3}$. Suppose $g^{-1}\left(\left\{q_{1}\right\}\right)=\left\{p_{3}\right\}, g^{-1}\left(\left\{q_{2}\right\}\right)=\left\{p_{0}\right\}$. Without loss of generality we can assume $q_{1}=\infty$. Then by considering $\tilde{g}=g-q_{2}$ instead of $g$, we can assume 
that $\tilde{g}$ misses $0, \infty$ and another point. Now we study the functional properties of $\tilde{g}$. Later, by using $g=a \tilde{g}+b, a, b \in \mathbb{C}$, we go back to the original $g$. Let $L=[1, \tau], \operatorname{Im} \tau>0$, be the lattice such that $T=\mathbb{C} / L$. So we are studying an elliptic function $\hat{g}$ such that if $\pi: \mathbb{C} \rightarrow T$ is the projection, then $\hat{g}=\tilde{g} \circ \pi$ and on $M, \tilde{g}$ misses $\{\infty, 0, q\}$. For simplicity, we will write $g$ instead of $\hat{g}$ below. By a translation in $\mathbb{C}$ if necessary, we can assume that $p_{3}=0, g(0)=\infty, g\left(p_{0}\right)=0$. By (2.10), $g\left(p_{1}\right)=g\left(p_{2}\right)=q$. Since $r=n=4$ and $k=1$, by (2.6), the total branching order of $g$ is $2 \times 4=8$. By Proposition 2.2, all the branch points are included in $\left\{0, p_{0}, p_{1}, p_{2}\right\}$ so we can assume without loss of generality that there are only two cases:

$\triangleright \quad$ CASE 1.0 and $p_{0}$ have branching order 3 and $p_{1}$ and $p_{2}$ have branching order 1 .

$\triangleright \quad$ CASE 2. 0 and $p_{0}$ have branching order $3, p_{1}$ has branching order 0 and $p_{2}$ has branching order 2 .

In fact, Case 2 is impossible. Since $p_{1}$ is not a branch point of $g$, by Proposition 1.1, $M$ has one catenoid end. By Corollary 2.2, this is impossible.

In Case $1, g$ is an elliptic function of order 4 whose only pole occurs at 0 and whose only zero occurs at $p_{0}$. We have the relationship ([11], Theorem 3 on page 7),

$$
4 p_{0} \equiv 0 \bmod L
$$

We will prove that Case 1 is also impossible.

Notice that Case 1 can be described as the case that the derivative $g^{\prime}$ of the Gauss map $g$ has three distinguished zero points, since we can always assume that $\{\infty\}$ is an omitted value of $g$ on $M$. First we prove several sublemmas. To prove them we need the Weierstrass elliptic function. For the details of elliptic function theory, please see [11], Chapter 1 . Let $L=[1, \tau]$ be a lattice. The Weierstrass elliptic function associated to $L$ is,

$$
\wp(z)=\frac{1}{z^{2}}+\sum_{\omega \in L, \omega \neq 0}\left[\frac{1}{(z-\omega)^{2}}-\frac{1}{\omega^{2}}\right]
$$

the Laurent expansion of $\wp$ at 0 is

$$
\wp(z)=\frac{1}{z^{2}}+3 s_{4} z^{2}+5 s_{6} z^{4}+\cdots
$$

It satisfies the differential equation

$$
\wp^{2}(z)=4\left(\wp(z)-e_{1}\right)\left(\wp(z)-e_{2}\right)\left(\wp(z)-e_{3}\right)
$$


where $e_{i}=\wp\left(\omega_{i}\right), \omega_{1}=1 / 2, \omega_{2}=(1+\tau) / 2$, and $\omega_{3}=\tau / 2$, are half periods of $L$. So $\wp^{\prime}(p)=0$ if and only if $p$ is a half period, since deg $\wp^{\prime}=3$, these are simple zeros of $\wp^{\prime}$. Moreover, $e_{i} \neq e_{j}$ for $i \neq j$, and

$$
e_{1}+e_{2}+e_{3}=0
$$

$e_{1} e_{2} e_{3}=0$ if and only if $L$ is equivalent to $[1, i]$ and $e_{2}=0, e_{1}=-e_{3} \neq 0$.

Sublemma 3.1. Suppose $X: M \rightarrow \mathbb{R}^{3}$ is a complete minimal immersion of a punctured torus $M=T-\left\{0, p_{0}, p_{1}, p_{2}\right\}$ of finite total curvature, whose Gauss map $g: M \rightarrow \mathbb{C} \cup\{\infty\}$ misses 3 values. Suppose the branching order of 0 and $p_{0}$ is 3 , and the branching order of $p_{1}$ and $p_{2}$ is 1 . Then:

(1) $T$ is a square torus. If $T$ is represented by $\mathbb{C} /[1, i]$, then;

(2) $p_{0}=(1+i) / 2, p_{1}=1 / 2, p_{2}=i / 2$, and;

(3) $g(z)=a \wp^{2}(z)+b, a, b \in \mathbb{C}, a \neq 0$.

Proof. Without loss of generality, we can assume $g(0)=\infty$. Letting $\tilde{g}=$ $g-g\left(p_{0}\right)$, we can assume $g\left(p_{0}\right)=0$. For simplicity, we will write $g$ instead of $\tilde{g}$. Since 0 is the only pole of $g$ in a fundamental parallelogram, the residue of $g$ at 0 must be 0 ([11], Theorem 1 on page 6). The Laurent expansion of $g$ at 0 must be

$$
g(z)=\frac{c_{-4}}{z^{4}}+\frac{c_{-3}}{z^{3}}+\frac{c_{-2}}{z^{2}}+c_{0}+c_{1} z+\cdots
$$

Without loss of generality, we can assume $c_{-4}=1$. Let $\wp$ be the Weierstrass function associated to the lattice $L$, by (3.12),

$$
g(z)-\wp^{2}(z)+\frac{c_{-3}}{2} \cdot \wp^{\prime}(z)-c_{-2} \wp(z)
$$

is an elliptic function without poles. Hence it is constant. Therefore,

$$
g(z)=\wp^{2}(z)+b \wp^{\prime}(z)+c \wp(z)+d .
$$

By (2.10), we have

$$
g\left(p_{1}\right)=g\left(p_{2}\right), \quad g^{\prime}\left(p_{1}\right)=g^{\prime}\left(p_{2}\right)=0
$$

Since $p_{1}, p_{2}$ are the only zeros of $g(z)-g\left(p_{1}\right)$ and they are both of order 2 , we get

$$
2\left(p_{1}+p_{2}\right) \equiv 0 \bmod L
$$


Since $g^{\prime}$ has a triple zero at $p_{0}$, two simple zeros at $p_{1}$ and $p_{2}$ and a pole of order 5 at 0 , we have

$$
3 p_{0}+p_{1}+p_{2} \equiv 0 \bmod L .
$$

Since $4 p_{0} \equiv 0 \bmod L$, we have

$$
-p_{0}+p_{1}+p_{2} \equiv 0 \bmod L \text {. }
$$

Multiplying (3.18) by 2 and using (3.16), we have

$$
2 p_{0} \equiv 0 \bmod L \text {. }
$$

Hence $p_{0}$ is a half period and $\wp^{\prime}\left(p_{0}\right)=0$. Since $g^{\prime}\left(p_{0}\right)=0$, we have

$$
0=g^{\prime}\left(p_{0}\right)=2 \wp^{\prime}\left(p_{0}\right) \wp\left(p_{0}\right)+b \wp^{\prime \prime}\left(p_{0}\right)+c \wp^{\prime}\left(p_{0}\right)=b \wp^{\prime \prime}\left(p_{0}\right) \text {. }
$$

Because $\wp^{\prime \prime}\left(p_{0}\right) \neq 0, b=0$. Thus we have

$$
g(z)=\wp^{2}(z)+c \wp(z)+d .
$$

Letting $\omega_{i}$ be the half periods mentioned before, we get

$$
g^{\prime}\left(\omega_{i}\right)=2 \wp\left(\omega_{i}\right) \wp^{\prime}\left(\omega_{i}\right)+c \wp^{\prime}\left(\omega_{i}\right)=0 .
$$

But the only zeros of $g^{\prime}$ are $p_{0}, p_{1}, p_{2}$, so we have $p_{0}=\omega_{k},\left\{p_{1}, p_{2}\right\}=\left\{\omega_{i}, \omega_{j}\right\}$, where $\{i, j, k\}=\{1,2,3\}$. Since $g^{\prime \prime}\left(\omega_{k}\right)=0$ and $\wp^{\prime}\left(\omega_{k}\right)=0$, we have

$$
0=g^{\prime \prime}\left(\omega_{k}\right)=2 \wp^{\prime 2}\left(\omega_{k}\right)+\wp^{\prime \prime}\left(\omega_{k}\right) \cdot\left[2 \wp\left(\omega_{k}\right)+c\right]=\wp^{\prime \prime}\left(\omega_{k}\right)\left[2 \wp\left(\omega_{k}\right)+c\right] .
$$

Since $\wp^{\prime \prime}\left(\omega_{k}\right) \neq 0,2 \wp\left(\omega_{k}\right)+c=0$. Hence $c=-2 \wp\left(\omega_{k}\right)=-2 e_{k}$. We get

$$
g(z)=\wp^{2}(z)-2 e_{k} \wp(z)+d .
$$

Since $g\left(\omega_{k}\right)=0$, we have

$$
e_{k}^{2}-2 e_{k}^{2}+d=0
$$

Hence $d=e_{k}^{2}$, thus

$$
g(z)=\wp^{2}(z)-2 e_{k} \wp(z)+e_{k}^{2}
$$

From $g\left(\omega_{i}\right)=g\left(\omega_{j}\right)$, we have

$$
e_{i}^{2}-2 e_{k} e_{i}+e_{k}^{2}=e_{j}^{2}-2 e_{k} e_{j}+e_{k}^{2}
$$

i.e., $e_{i}\left(e_{i}-2 e_{k}\right)=e_{j}\left(e_{j}-2 e_{k}\right)$. By (3.14), $-2 e_{k}=2\left(e_{i}+e_{j}\right)$. So we get

$$
e_{i}\left(3 e_{i}+2 e_{j}\right)=e_{j}\left(2 e_{i}+3 e_{j}\right)
$$

i.e., $3 e_{i}^{2}=3 e_{j}^{2}$. It follows that $e_{i}= \pm e_{j}$. Since $e_{i} \neq e_{j}$ so $e_{i}=-e_{j}$. Hence by (3.14) we know that $e_{k}=0$, and by (3.21),

$$
g(z)=\wp^{2}(z) \text {. }
$$

But $e_{k}=0$ only when $L=[1, i]$ and $\omega_{k}=(1+\tau) / 2=\omega_{2}$, as mentioned before. The proof of this sublemma is completed. 
Sublemma 3.2. Suppose $M=\mathbb{C} /[1, i]-\{0,1 / 2, i / 2,(1+i) / 2\}$ and $\wp$ is the associated Weierstrass function. Define

$$
\left\{\begin{array}{l}
\varphi=\left[\wp^{2}(z) / \wp^{\prime 2}(z)\right] d z \\
\psi=\left[\wp^{4}(z) / \wp^{\prime 2}(z)\right] d z \\
\eta=d z / \wp^{\prime 2}(z) .
\end{array}\right.
$$

Let $\gamma_{1}$ be the line segment in $S$ from $\frac{1}{4}+i$ to $\frac{1}{4}, \gamma_{2}$ from $\frac{3}{4} i$ to $1+\frac{3}{4} i$. Then

$$
\begin{gathered}
\int_{\gamma_{1}} \varphi=i B, \quad \int_{\gamma_{2}} \varphi=B, \quad \int_{\gamma_{1}} \psi=i A \\
\int_{\gamma_{2}} \psi=A, \quad \int_{\gamma_{1}} \eta=i C, \quad \int_{\gamma_{2}} \eta=C,
\end{gathered}
$$

where $A, B, C \in \mathbb{R}$ and $B, C$ are not zero.

Proof. Let $\zeta$ be the Weierstrass Zeta function: $\zeta^{\prime}(z)=-\wp(z)$, then a known fact is (see [1] or [11])

$$
\zeta\left(\frac{1}{4}+i\right) \neq \zeta\left(\frac{1}{4}\right)
$$

Let $\bar{z}$ be the complex conjugate of $z$. Consider the following four reflections in the complex plane $\mathbb{C}$. $R(z)=-i \bar{z}+1+i, K(z)=1-\bar{z}, Q(z)=i \bar{z}$ and $N(z)=\bar{z}+i$. Let $S$ be the square $\{z=x+i y \mid 0 \leq x \leq 1,0 \leq y \leq 1\}$. Clearly these reflections leave $S$ invariant and

$$
Q=K \circ R \circ K, \quad N=R \circ K \circ R
$$

We see that

$$
R^{*} d z=-i d \bar{z}, \quad K^{*} d z=-d \bar{z}
$$

Since $\wp$ satisfies (see [1])

$$
\wp \circ R(z)=-\overline{\wp(z)}, \quad \wp \circ K(z)=\overline{\wp(z)} .
$$

By (3.13), noticing that now $e_{2}=0$ and $e_{1}=-e_{3}$, we have

$$
\wp^{\prime 2} \circ R(z)=-\overline{\wp^{\prime 2}(z)}, \quad \wp^{\prime 2} \circ K(z)=\overline{\wp^{\prime 2}(z)}
$$


Thus for the three forms defined in (3.23), we get

$$
\begin{gathered}
R^{*} \varphi=\frac{\wp^{2} \circ R(z)}{\wp^{\prime 2} \circ R(z)} R^{*} d z=-i \overline{\left(\frac{\wp^{2}(z)}{-\wp^{\prime 2}(z)} d z\right)}=i \bar{\varphi}, \quad R^{*} \bar{\varphi}=-i \varphi \\
R^{*} \psi=\frac{\wp^{4} \circ R(z)}{\wp^{\prime 2} \circ R(z)} R^{*} d z=-i \overline{\left(\frac{\wp^{4}(z)}{-\wp^{\prime 2}(z)} d z\right)}=i \bar{\psi}, \quad R^{*} \bar{\psi}=-i \psi . \\
R^{*} \eta=\frac{R^{*} d z}{\wp^{\prime 2} \circ R(z)}=i \bar{\eta}, \quad R^{*} \bar{\eta}=-i \eta .
\end{gathered}
$$

Similarly we have,

$$
\begin{aligned}
& K^{*} \varphi=-\bar{\varphi}, \quad K^{*} \bar{\varphi}=-\varphi, \quad K^{*} \psi=-\bar{\psi}, \\
& K^{*} \bar{\psi}=-\psi, \quad K^{*} \eta=-\bar{\eta}, \quad K^{*} \bar{\eta}=-\eta .
\end{aligned}
$$

By (3.25) we have

$$
\begin{aligned}
& Q^{*} \varphi=K^{*} \circ R^{*} \circ K^{*} \varphi=-i \bar{\varphi}, \quad Q^{*} \bar{\varphi}=i \varphi \\
& Q^{*} \psi=-i \bar{\psi}, \quad Q^{*} \bar{\psi}=i \psi, \quad Q^{*} \eta=-i \bar{\eta}, \quad Q^{*} \bar{\eta}=i \eta, \\
& N^{*} \varphi=\bar{\varphi}, \quad N^{*} \bar{\varphi}=\varphi, \quad N^{*} \psi=\bar{\psi}, \quad N^{*} \bar{\psi}=\psi, \\
& N^{*} \eta=\bar{\eta}, \quad N^{*} \bar{\eta}=\eta .
\end{aligned}
$$

Let $\gamma_{1}$ and $\gamma_{2}$ be as defined in the statement of the sublemma, $\gamma_{3}$ be the line segment from $\frac{3}{4}+i$ to $\frac{3}{4}$ and $\gamma_{4}$ from $\frac{1}{4} i$ to $1+\frac{1}{4} i$. By the definition of $R$, $Q, N, K$, we see that

$$
\begin{gathered}
R\left(\gamma_{1}\right)=\gamma_{2}, \quad R\left(\gamma_{3}\right)=\gamma_{4}, \quad K\left(\gamma_{1}\right)=\gamma_{3}, \\
Q\left(\gamma_{2}\right)=-\gamma_{3}, \quad Q\left(\gamma_{1}\right)=-\gamma_{4}, \quad N\left(\gamma_{2}\right)=\gamma_{4} .
\end{gathered}
$$

Let $\int_{\gamma_{1}} \varphi=x+y i$ and $\int_{\gamma_{2}} \varphi=u+v i$. Then we can compute that

$$
x+y i=\int_{\gamma_{1}} \varphi=\int_{R\left(\gamma_{2}\right)} \varphi=\int_{\gamma_{2}} R^{*} \varphi=i \int_{\gamma_{2}} \bar{\varphi}=i \overline{\int_{\gamma_{2}} \varphi}=i(u-v i) .
$$

Hence we have $x+y i=i(u-v i)=v+u i$ so $x=v$ and $y=u$. Now using other symmetries, we get

$$
\begin{aligned}
x+y i & =\int_{\gamma_{1}} \varphi=\int_{K\left(\gamma_{3}\right)} \varphi=\int_{\gamma_{3}} K^{*} \varphi=-\int_{\gamma_{3}} \bar{\varphi} \\
& =\int_{Q\left(\gamma_{2}\right)} \bar{\varphi}=\int_{\gamma_{2}} Q^{*} \bar{\varphi}=i \int_{\gamma_{2}} \varphi=i(u+v i)=-v+u i .
\end{aligned}
$$


Hence $v=x=-v=0$. Letting $B=y=u \in \mathbb{R}$, we get

$$
\int_{\gamma_{1}} \varphi=i B, \quad \int_{\gamma_{2}} \varphi=B .
$$

Since

$$
\begin{aligned}
i B=\int_{\gamma_{1}} \varphi & =-\int_{1 / 4}^{1 / 4+i} \frac{\wp^{2}(z)}{\wp^{2}(z)} d z \\
& =-\frac{1}{4} \int_{1 / 4}^{1 / 4+i} \frac{\wp(z)}{\wp^{2}(z)-e_{1}^{2}} d z \\
& =-\frac{1}{8} \int_{1 / 4}^{1 / 4+i}\left(\frac{1}{\wp(z)-e_{1}}+\frac{1}{\wp(z)+e_{1}}\right) d z \\
& =-\frac{1}{16 e_{1}^{2}}\left\{-\left[e_{1} z+\zeta(z)+\frac{\wp^{\prime}(z)}{2\left(\wp(z)-e_{1}\right)}\right]\right. \\
& =\frac{1}{16 e_{1}^{2}}\left[2 \zeta(z)+\frac{\wp^{\prime}(z)}{2\left(\wp(z)-e_{1}\right)}+\frac{\wp^{\prime}(z)}{2\left(\wp(z)+e_{1}\right)}\right]_{1 / 4}^{1 / 4+i} \\
& \left.=\frac{\zeta(1 / 4+i)-\zeta(1 / 4)}{8 e_{1}^{2}},\right\}_{1 / 4}^{1 / 4+i}
\end{aligned}
$$

it follows from (3.24) that $B \neq 0$. All these integrals can be found in ([2], page 311 ). Notice that here we have $e_{1}=-e_{3} \neq 0$ and $e_{2}=0$. In the same manner we can prove that

$$
\int_{\gamma_{1}} \eta=i C, \quad \int_{\gamma_{2}} \eta=C
$$

and

$$
\int_{\gamma_{1}} \psi=i A, \quad \int_{\gamma_{2}} \psi=A,
$$

where $A, C \in \mathbb{R}$. Furthermore,

$$
\begin{aligned}
\int_{\gamma_{1}} \eta & =-\int_{1 / 4}^{1 / 4+i} \eta=-\int_{1 / 4}^{1 / 4+i} \frac{d z}{\wp^{\prime 2}(z)} \\
& =-\frac{1}{4} \int_{1 / 4}^{1 / 4+i} \frac{d z}{\wp(z)\left(\wp^{2}(z)-e_{1}^{2}\right)}=
\end{aligned}
$$




$$
\begin{aligned}
& =\frac{1}{8 e_{1}^{2}} \int_{1 / 4}^{1 / 4+i}\left(\frac{2}{\wp(z)}-\frac{1}{\wp(z)-e_{1}}-\frac{1}{\wp(z)+e_{1}}\right) d z \\
& =\frac{1}{16 e_{1}^{4}}\left\{4\left[\zeta(z)+\frac{\wp^{\prime}(z)}{2 \wp(z)}\right]+\left[e_{1} z+\zeta(z)+\frac{\wp^{\prime}(z)}{2\left(\wp(z)-e_{1}\right)}\right]\right. \\
& \left.+\left[-e_{1} z+\zeta(z)+\frac{\wp^{\prime}(z)}{2\left(\wp(z)+e_{1}\right)}\right]\right\}_{1 / 4}^{1 / 4+i} \\
& =\frac{1}{16 e_{1}^{4}}\left\{6 \zeta(z)+\left[2 \frac{\wp^{\prime}(z)}{\wp(z)}+\frac{\wp^{\prime}(z)}{2\left(\wp(z)-e_{1}\right)}+\frac{\wp^{\prime}(z)}{2\left(\wp(z)+e_{1}\right)}\right]\right\}_{1 / 4}^{1 / 4+i} \\
& =\frac{3}{8 e_{1}^{4}}\left[\zeta\left(\frac{1}{4}+i\right)-\zeta\left(\frac{1}{4}\right)\right]=\frac{3 i B}{e_{1}^{2}} \neq 0 .
\end{aligned}
$$

Hence the sublemma is proved.

Sublemma 3.3. Suppose $M=\mathbb{C} /[1, i]-\{0,1 / 2, i / 2,(1+i) / 2\}$ and $g(z)=$ $a \wp^{2}(z)+b, a, b \in \mathbb{C}, a \neq 0$. Then $g$ is not the Gauss map of a complete, minimal immersion in $\mathbb{R}^{3}$ that is well defined on $M$. Therefore, Case 1 is impossible.

Proof. If $g$ is the Gauss map of a complete minimal immersion of $M$, then since $\operatorname{deg} g=4, C(M)=-16 \pi$. Since on $M=T-\{0,1 / 2, i / 2,(1+i) / 2\}, g$ misses three values $\left\{\infty, b, a e_{1}^{2}+b\right\}$, where $e_{1}=\wp(1 / 2)=-\wp(i / 2)$, it follows from Proposition 2.1 that every end of $M$ is embedded. Hence by Proposition 1.1 the divisors of $g, 1 / \wp^{\prime 2}$ and the possible 1 -form $\eta=f(z) d z$ are as follows:

$$
\left[\begin{array}{ccccc} 
& 0 & \frac{1}{2} & \frac{i}{2} & \frac{1+i}{2} \\
g & \infty^{4} & & & \\
f & 0^{6} & \infty^{2} & \infty^{2} & \infty^{2} \\
\frac{1}{\wp^{\prime 2}} & 0^{6} & \infty^{2} & \infty^{2} & \infty^{2}
\end{array}\right]
$$

Thus $f \wp^{\prime 2}=c \neq 0$ is a constant, and $f=c / \wp^{\prime 2}$.

Clearly $\gamma_{1}$ and $\gamma_{2}$ in Sublemma 3.2 represent the two generators of the fundamental group of $T$.

Now consider the three differential forms in the Enneper-Weierstrass representation.

$$
\omega_{1}=\frac{c}{2}\left[\frac{1-\left(a \wp^{2}(z)+b\right)^{2}}{\wp^{\prime 2}(z)}\right] d z
$$




$$
\begin{aligned}
& \omega_{2}=\frac{i c}{2}\left[\frac{1+\left(a \wp^{2}(z)+b\right)^{2}}{\wp^{2}(z)}\right] d z, \\
& \omega_{3}=c\left[\frac{a \wp^{2}(z)+b}{\wp^{2}(z)}\right] d z .
\end{aligned}
$$

By (3.23), (3.30), (3.31), and (3.32),

$$
\begin{aligned}
\omega_{1} & =\frac{c}{2}\left[\left(1-b^{2}\right) \eta-a^{2} \psi-2 a b \varphi\right], \\
\omega_{2} & =\frac{i c}{2}\left[\left(1+b^{2}\right) \eta+a^{2} \psi+2 a b \varphi\right], \\
\omega_{3} & =c(a \varphi+b \eta) .
\end{aligned}
$$

They give a well-defined minimal immersion if and only if

$$
\operatorname{Re} \int_{\gamma_{i}} \omega_{j}=0, \quad i=1,2 ; j=1,2,3
$$

To make $\omega_{3}$ have no real periods, we need

$$
\operatorname{Re} \int_{\gamma_{1}} \omega_{3}=0, \quad \operatorname{Re} \int_{\gamma_{2}} \omega_{3}=0
$$

By Sublemma 3.2,

$$
\begin{gathered}
0=\operatorname{Re} \int_{\gamma_{1}} \omega_{3}=\operatorname{Re} c\left(a \int_{\gamma_{1}} \varphi+b \int_{\gamma_{1}} \eta\right) \\
=\operatorname{Re} c(a B i+b C i)=-\operatorname{Im} c(a B+b C) \\
0=\operatorname{Re} \int_{\gamma_{2}} \omega_{3}=\operatorname{Rec}\left(a \int_{\gamma_{2}} \varphi+b \int_{\gamma_{2}} \eta\right)=\operatorname{Re} c(a B+b C)
\end{gathered}
$$

Thus $a B+b C=0$, because $c \neq 0$.

Since $a \neq 0, B \neq 0, C \neq 0, b=-(B / C) a \neq 0$. So for any non-zero $a \in \mathbb{C}$, then $b$ must be equal to $-(B / C) a$. Hence we get

$$
\begin{aligned}
& \omega_{1}=\frac{c}{2}\left\{\left[1-\left(\frac{B}{C}\right)^{2} a^{2}\right] \eta-a^{2} \psi+2\left(\frac{B}{C}\right) a^{2} \varphi\right\}, \\
& \omega_{2}=\frac{i c}{2}\left\{\left[1+\left(\frac{B}{C}\right)^{2} a^{2}\right] \eta+a^{2} \psi-2\left(\frac{B}{C}\right) a^{2} \varphi\right\} .
\end{aligned}
$$


The integrals are

$$
\begin{aligned}
& 2 \int_{\gamma_{1}} \omega_{1}=i c\left\{\left[1-\left(\frac{B}{C}\right)^{2} a^{2}\right] C-a^{2} A+2\left(\frac{B}{C}\right) a^{2} B\right\} \\
& 2 \int_{\gamma_{2}} \omega_{1}=c\left\{\left[1-\left(\frac{B}{C}\right)^{2} a^{2}\right] C-a^{2} A+2\left(\frac{B}{C}\right) a^{2} B\right\}=-i 2 \int_{\gamma_{1}} \omega_{1} \\
& 2 \int_{\gamma_{1}} \omega_{2}=-c\left\{\left[1+\left(\frac{B}{C}\right)^{2} a^{2}\right] C+a^{2} A-2\left(\frac{B}{C}\right) a^{2} B\right\} \\
& 2 \int_{\gamma_{2}} \omega_{2}=-i c\left\{\left[1+\left(\frac{B}{C}\right)^{2} a^{2}\right] C+a^{2} A-2\left(\frac{B}{C}\right) a^{2} B\right\}=-i 2 \int_{\gamma_{1}} \omega_{2}
\end{aligned}
$$

Hence requiring $\operatorname{Re} \int_{\gamma_{i}} \omega_{j}=0$, for $1 \leq i, j \leq 2$, is equivalent to requiring

$$
\begin{aligned}
& \left(1-\left(\frac{B}{C}\right)^{2} a^{2}\right) C-a^{2} A+2\left(\frac{B}{C}\right) a^{2} B=0 \\
& \left(1+\left(\frac{B}{C}\right)^{2} a^{2}\right) C+a^{2} A-2\left(\frac{B}{C}\right) a^{2} B=0
\end{aligned}
$$

Adding (3.34) and (3.35), we get $2 C=0$. But we know that $C \neq 0$ from Sublemma 3.2. By Sublemma 3.1, Case 1 implies that $g=a \wp^{2}+b$, thus we have completed the proof of this sublemma.

Lemma 3.2. There does not exist a genus-1 complete, immersed minimal surface with total curvature $-16 \pi$ whose Gauss map misses three values.

Proof. By the discussion before Sublemma 3.1, there only two possibilities, namely, Case 1 and Case 2. We have already ruled out Case 2, now Sublemma 3.3 rules out Case 1 . So the proof is completed.

3.2. The Genus-2 Case. To consider the genus-2 case we need some notations of divisors and Riemann-Roch theorem, we follow the notations in [5]. Let $M_{k}$ be a genus- $k$ compact Riemann surface. A divisor on $M_{k}$ is

$$
D=\prod_{P \in M_{k}} P^{\alpha_{P}},
$$

where $\alpha_{P}$ are integers, only finite number of them are not zero. Define

$$
\operatorname{deg} D=\sum_{P \in M_{k}} \alpha_{P},
$$




$$
D^{-1}=\prod_{P \in M_{k}} P^{-\alpha_{P}}
$$

Let

$$
D^{\prime}=\prod_{P \in M_{k}} P^{\beta_{P}}
$$

then $D \geq D^{\prime}$ if and only if $\alpha_{P} \geq \beta_{P}$ for all $P \in M_{k}$. The product $D D^{\prime}$ of two divisors as in (3.36) and (3.37) is defined as

$$
D D^{\prime}=\prod_{P \in M_{k}} P^{\alpha_{P}+\beta_{P}}
$$

Clearly all the divisors consist of a group. Let

$$
\mathcal{K}\left(M_{k}\right)=\left\{f: f \text { is a meromorphic function on } M_{k}\right\}
$$

$\mathcal{K}\left(M_{k}\right)$ is an algebra over $\mathbb{C}$. For a $f \in \mathcal{K}\left(M_{k}\right)$, we can define the divisor $(f)$ as follows:

$$
(f)=\prod_{P \in M_{k}} P^{\operatorname{ord}_{P} f}
$$

and the polar divisor of $f$

$$
f^{1}(\infty)=\prod_{P \in M_{k}} P^{\max \left(-\operatorname{ord}_{P} f, 0\right)}
$$

For a meromorphic 1-form $\omega$, define

$$
(\omega)=\prod_{P \in M_{k}} P^{\mathrm{ord}_{P} \omega} .
$$

Then $\operatorname{deg}(f)=0$, and $\operatorname{deg}(\omega)=2 k-2$. Let $D$ be any divisor, define

$$
L(D)=\left\{f \in \mathcal{K}\left(M_{k}\right):(f) \geq D\right\}
$$

$$
\Omega(D)=\left\{\omega: \omega \text { is a meromorphic 1-form on } M_{k},(\omega) \geq D\right\} .
$$

The holomorphic 1-forms on $M_{k}$ form a $k$-dimensional vector space. Any two meromorphic 1-forms $\omega_{1}$ and $\omega_{2}$ define a meromorphic function

$$
f=\frac{\omega_{1}}{\omega_{2}}
$$


Denote that $r(D)=\operatorname{dim} L(D), i(D)=\operatorname{dim} \Omega(D)$, then the Riemann-Roch theorem tells us

$$
r\left(D^{-1}\right)=\operatorname{deg}(D)-k+1+i(D) .
$$

When $\operatorname{deg} D \geq 2 k-1, i(D)=0$, so

$$
r\left(D^{-1}\right)=\operatorname{deg} D-k+1 .
$$

Since any genus-2 Riemann surface is a hyperelliptic surface, there is a degree-2 meromorphic function $z: M_{2} \rightarrow \mathbb{C} \cup\{\infty\}$. The function

$$
w=\sqrt{\prod_{i=1}^{6}\left(z-c_{i}\right)}
$$

is a well defined meromorphic function and it has degree 6 . Where $c_{i} \neq c_{j}$, for $i \neq j$, are the branch points of the function $z$, i.e., $d z\left(c_{i}\right)=0$, they are also called Weierstrass points. Without loss of generality, we can assume that $c_{i} \neq \infty$. Let $\left\{Q_{1}, Q_{2}\right\}=z^{-1}(\{\infty\})$, then $w$ has a pole of multiplicity 3 at each of $Q_{1}, Q_{2}$. A general point of $M_{2}$ is denoted as $p=(z(p), w(z(p)))$. There is a conformal involution $J: M_{2} \rightarrow M_{2}$,

$$
J(p)=J((z(p), w(z(p))))=(z(p),-w(z(p))),
$$

the only fixed points of $J$ are the Weierstrass points. A basis of holomorphic 1 -forms on $M_{2}$ is given by

$$
\left\{\frac{d z}{w}, \frac{z d z}{w}\right\}
$$

$d z / w$ has two simple zeros at $Q_{1}$ and $Q_{2}, z d z / w$ has two simple zeros at $(0, w(0))$ and $(0,-w(0))$ if $w(0) \neq 0$, otherwise, it has a double zero at $(0,0)$.

Lemma 3.3. There exist no genus-2 immersed complete minimal surface $M$ with total curvature $-16 \pi$, whose Gauss map misses 3 values.

Proof. By Lemma 3.1, if there exists such a surface, it has only three embedded flat ends. And its Gauss map $g$ has degree 4. If $X: M_{2}-\left\{p_{1}, p_{2}, p_{3}\right\}$ is such an immersion, then by a rotation if necessary, we can assume that the Gauss map $g$ has pole at $p_{1}$. The related divisors of $g$ are, $g^{-1}(\infty)=D^{\prime}=p_{1}^{4}$, and $\left(g-g\left(p_{i}\right)\right)=p_{1}^{-4} p_{i}^{4}, i=2,3$, since $g$ misses three values on $M_{2}-\left\{p_{1}, p_{2}, p_{3}\right\}$, and $\operatorname{deg} g=4$. The total branching order among $\left\{p_{1}, p_{2}, p_{3}\right\}$ is 9 . The total branching order of $g$ on $M_{2}$ is, by (2.6)

$$
n_{0}=2(4-1+2)=10
$$


hence $g$ has only one branch point on $M_{2}-\left\{p_{1}, p_{2}, p_{3}\right\}$. We claim that $p_{i}$ 's are not Weierstrass points. In fact, if, say, $p_{1}$ is a Weierstrass point, then say, $z\left(p_{1}\right)=c_{1}$. By the Riemann-Roch theorem, $r\left(D^{\prime-1}\right)=4-1=3$. Then since $c_{1}$ is a double zero of $z-c_{1}$,

$$
\left(1, \frac{1}{z-c_{1}}, \frac{1}{\left(z-c_{1}\right)^{2}}\right)
$$

is a basis of $L\left(D^{\prime-1}\right)$, where 1 means the constant function of value 1 . Thus the Gauss map of $M_{2}-\left\{p_{1}, p_{2}, p_{3}\right\}$ will be

$$
\begin{aligned}
g & =a+\frac{b}{z-c_{1}}+\frac{c}{\left(z-c_{1}\right)^{2}} \\
d g & =\left(\frac{b}{z-c_{1}}+\frac{c}{\left(z-c_{1}\right)^{2}}\right)^{\prime} d z .
\end{aligned}
$$

Hence $g$ has branch points of $c_{i}, i=2, \ldots, 6$, contradicts that $g$ has only one branch point on $M_{2}-\left\{p_{1}, p_{2}, p_{3}\right\}$. If $\left\{Q_{1}, Q_{2}\right\} \not \subset\left\{p_{1}, p_{2}, p_{3}\right\}$, we can always select $p_{1}, p_{2}$, and $p_{3}$ such that $z\left(p_{2}\right) \neq z\left(p_{3}\right)$, and $z\left(p_{1}\right)$ is finite, otherwise we just change the role of $p_{1}, p_{2}$, and $p_{3}$. If $\left\{Q_{1}, Q_{2}\right\} \subset\left\{p_{1}, p_{2}, p_{3}\right\}$, we choose $p_{2}=Q_{1}$ and $p_{3}=Q_{2}$. By Proposition 1.1 and Lemma 3.1, if there exists such a surface, then there should be a meromorphic 1-form $\eta$ with the divisor

$$
D=(\eta)=p_{1}^{6} p_{2}^{-2} p_{3}^{-2}
$$

See the table (3.38) below to get a clearer picture. Then

$$
r\left(D^{-1}\right)=\operatorname{deg}(D)-2+1+i(D)=1+i(D)=2 .
$$

There are two linear independent functions $f_{1}$ and $f_{2}$, such that

$$
\operatorname{span}\left(f_{1}, f_{2}\right)=L\left(D^{-1}\right)
$$

Now $f_{i} \eta$ is a holomorphic 1 -form, $i=1,2$, so

$$
A\left(\begin{array}{c}
f_{1} \\
f_{2}
\end{array}\right) \eta=\left(\begin{array}{c}
d z / w \\
z d z / w
\end{array}\right)
$$

where $A$ is a two by two non-degenerate matrix, hence we can choose

$$
f_{1} \eta=\frac{d z}{w}, \quad f_{2} \eta=\frac{z d z}{w} .
$$


So

$$
\left(f_{1}\right)=\left(\frac{d z}{w \eta}\right)=p_{1}^{-6} p_{2}^{2} p_{3}^{2} Q_{1}^{1} Q_{2}^{1}
$$

Let $f=f_{1}^{-1}$, then

$$
(f)=p_{1}^{6} p_{2}^{-2} p_{3}^{-2} Q_{1}^{-1} Q_{2}^{-1} .
$$

To look at these divisors more clearly, we make a table, let $g_{2}=g-g\left(p_{2}\right)$, $g_{3}=g-g\left(p_{3}\right)$, and remember that $f_{1}=d z / w \eta, f=f_{1}^{-1}$.

$$
\left[\begin{array}{cccccc} 
& p_{1} & p_{2} & p_{3} & Q_{1} & Q_{2} \\
g_{2} & \infty^{4} & 0^{4} & & & \\
g_{3} & \infty^{4} & & 0^{4} & & \\
g_{2} g_{3} & \infty^{8} & 0^{4} & 0^{4} & & \\
\eta & 0^{6} & \infty^{2} & \infty^{2} & & \\
d z / w & & & & 0^{1} & 0^{1} \\
f_{1} & \infty^{6} & 0^{2} & 0^{2} & 0^{1} & 0^{1} \\
f & 0^{6} & \infty^{2} & \infty^{2} & \infty^{1} & \infty^{1} \\
f g_{2} g_{3} & \infty^{2} & 0^{2} & 0^{2} & \infty^{1} & \infty^{1}
\end{array}\right]
$$

Now let

$$
h=\left(f g_{2} g_{3}\right)^{-1}
$$

then since $g\left(p_{2}\right) \neq g\left(p_{3}\right)$ and

$$
\left(f g_{2} g_{3}\right)=p_{1}^{-2} p_{2}^{2} p_{3}^{2} Q_{1}^{-1} Q_{2}^{-1},
$$

$$
(h)=p_{1}^{2} p_{2}^{-2} p_{3}^{-2} Q_{1}^{1} Q_{2}^{1} .
$$

By a translation $u=z-z\left(p_{1}\right)$, we can assume that $z\left(p_{1}\right)=0$. Remember that $z$ has simple poles at $Q_{1}$ and $Q_{2}$,

$$
\begin{gathered}
(z h)=p_{1}^{3}\left(J\left(p_{1}\right)\right)^{1} p_{2}^{-2} p_{3}^{-2}, \\
\left(z^{2} h\right)=p_{1}^{4}\left(J\left(p_{1}\right)\right)^{2} p_{2}^{-2} p_{3}^{-2} Q_{1}^{-1} Q_{2}^{-1} .
\end{gathered}
$$

$h, z h$, and $z^{2} h$ are not constant functions, $\operatorname{deg} h \leq 4, \operatorname{deg} z h=4$. Let $D_{2}=$ $p_{2}^{2} p_{3}^{2} Q_{1}^{1} Q_{2}^{1}$, then $z, h, z h, z^{2} h \in L\left(D_{2}^{-1}\right)$. Since $k=2, \operatorname{deg}\left(D_{2}\right)=6>3=2 k-1$, $r\left(\left(D_{2}\right)^{-1}\right)=6-1=5$.

We claim

$$
\operatorname{span}\left(1, z, h, z h, z^{2} h\right)=L\left(\left(D_{2}\right)^{-1}\right)
$$


Since $r\left(\left(D_{2}\right)^{-1}\right)=5$, we only need to prove that $\left(1, z, h, z h, z^{2} h\right)$ are linearly independent. If not, then there are complex numbers $(a, b, c, d, e) \neq(0,0,0,0,0)$, such that

$$
0 \equiv a+b z+\left(c+d z+e z^{2}\right) h
$$

it must be

$$
h=-\frac{a+b z}{c+d z+e z^{2}},
$$

then $h(p)=h(J(p))$ and $h$ has poles at $J\left(p_{2}\right)$ and $J\left(p_{3}\right)$. Since $p_{i}$ 's are not Weierstrass points, if $\left\{Q_{1}, Q_{2}\right\} \not \subset\left\{p_{1}, p_{2}, p_{3}\right\}$, then $z\left(p_{2}\right) \neq z\left(p_{3}\right), h$ has at least 6 poles, which is impossible. If $\left\{Q_{1}, Q_{2}\right\} \subset\left\{p_{1}, p_{2}, p_{3}\right\}$, then by (3.39), $(h)=p_{1}^{2} Q_{1}^{-1} Q_{2}^{-1}, h$ has double zero at $p_{1}$ and $J\left(p_{1}\right), p_{1}$ is not a Weierstrass point, contradicts $\operatorname{deg} h=2$. Then it must be $a=b=c=d=e=0$, $\left(1, z, h, z h, z^{2} h\right)$ are linearly independent. Since $f \in L\left(\left(D_{2}\right)^{-1} p_{1}^{6}\right) \subset L\left(\left(D_{2}\right)^{-1}\right)$, there are $(a, b, c, d, e) \neq(0,0,0,0,0)$, such that

$$
f=a+b z+c h+d z h+e z^{2} h .
$$

Since $p_{1}$ is not a branch point of $z$, we can take $z$ as a local coordinate at $p_{1}$, then write $h\left(p_{1}\right)$ as $h(0)$, etcetera:

$$
\begin{aligned}
A\left(\begin{array}{l}
a \\
b \\
c \\
d \\
e
\end{array}\right) & =\left(\begin{array}{llccc}
1 & 0 & h(0) & 0 & 0 \\
0 & 1 & h^{\prime}(0) & h(0) & 0 \\
0 & 0 & h^{\prime \prime}(0) & 2 h^{\prime}(0) & 2 h(0) \\
0 & 0 & h^{\prime \prime \prime}(0) & 3 h^{\prime \prime}(0) & 4 h^{\prime}(0) \\
0 & 0 & h^{(4)}(0) & 4 h^{\prime \prime \prime}(0) & 12 h^{\prime \prime}(0) \\
0 & 0 & h^{(5)}(0) & 5 h^{(4)}(0) & 20 h^{\prime \prime \prime}(0)
\end{array}\right)\left(\begin{array}{l}
a \\
b \\
c \\
d \\
e
\end{array}\right) \\
& =\left(\begin{array}{c}
f(0) \\
f^{\prime}(0) \\
f^{\prime \prime}(0) \\
f^{\prime \prime \prime}(0) \\
f^{(4)}(0) \\
f^{(5)}(0)
\end{array}\right)=\left(\begin{array}{l}
0 \\
0 \\
0 \\
0 \\
0 \\
0
\end{array}\right),
\end{aligned}
$$

since $f$ has zero of multiplicity 6 at $p_{1}$. Thus it must be $\operatorname{rank} A \leq 4$. Now remember that $h(0)=h^{\prime}(0)=0$, in fact,

Thus $\operatorname{rank} A \leq 4$ if and only if

$$
h \in L\left(p_{1}^{2} p_{2}^{-2} p_{3}^{-2} Q_{1}^{1} Q_{2}^{1}\right) .
$$

$$
h^{\prime \prime}(0)=h^{\prime \prime \prime}(0)=0 .
$$

Thus if rank $A \leq 4, z h$ has a zero of multiplicity 5 at $p_{1}$, which contradicts that $\operatorname{deg} z h=4$. This contradiction proves that $g$ and $\eta$ cannot co-exist, hence there are no such surfaces. The proof is complete. 
Combining Lemmas 3.1, 3.2, and 3.3, we have proved Theorem 3.1.

\section{Singly Periodic Minimal Surfaces Whose Gauss Map Misses Three Val-} ues. Note that in (3.34) if we choose $c \in \mathbb{R}$, and then let $a^{2}$ be a non-zero real number such that

$$
\left(1-\left(\frac{B}{C}\right)^{2} a^{2}\right) C-a^{2} A+2\left(\frac{B}{C}\right) a^{2} B=0
$$

then we will have

$$
\int_{\gamma_{2}} \omega_{1}=0
$$

and

$$
\int_{\gamma_{2}} \omega_{2} \in i \mathbb{R}
$$

So we get a singly periodic minimal surface whose Gauss map misses three values. A fundamental piece of it is a torus with four punctures, which has total Gauss curvature $-16 \pi$. Its only period is

$$
\int_{\gamma_{1}} \omega_{2}=-\frac{c}{2}\left\{\left[1+\left(\frac{B}{C}\right)^{2} a^{2}\right] C+a^{2} A-2\left(\frac{B}{C}\right) a^{2} B\right\}
$$

By choosing $a^{2}$ such that

$$
\left(1+\left(\frac{B}{C}\right)^{2} a^{2}\right) C+a^{2} A-2\left(\frac{B}{C}\right) a^{2} B=0
$$

we get another singly periodic minimal surface with the same property and its only period is

$$
\int_{\gamma_{2}} \omega_{1}=\frac{c}{2}\left\{\left[1-\left(\frac{B}{C}\right)^{2} a^{2}\right] C-a^{2} A+2\left(\frac{B}{C}\right) a^{2} B\right\}
$$

Acknowledgments. Part of this paper is based on the author's thesis at the University of Massachusetts, Amherst, directed by Professor David Hoffman whom the author thanks heartily. 


\section{REFERENCES}

[1] M. Abromovitz \& I. Stegun, Handbook of Mathematical Functions, Chapter 18, Advanced Mathematics, Dover Publications, Inc., New York, 1972.

[2] P. F. Byrd \& M. D. Friedman, Handbook of Elliptic Integrals for Engineers and Physicists, Springer-Verlag, Berlin, 1954.

[3] M. Callahan, D. Hoffman 6 W. Meeks III, Embedded minimal surfaces with an infinite number of ends, Invent. Math. 96 (1989), 459-505.

[4] Y. FANG, Complete minimal surfaces of finite total curvatures, Ph.D thesis, Univ. Mass. Amherst, 1990.

[5] H. M. FARKAS \& I. KRA, Riemann Surfaces, Springer-Verlag, New York, 1980.

[6] H. Fujimoto, On the number of exceptional values of the Gauss maps of minimal surfaces, J. Math. Soc. Japan 40 (1988), 235-247.

[7] F. GACKSTÄtTeR, Über abelsche Minimalflächen, Math. Nachr. 74 (1976), 157-165.

[8] L. Jorge \& W. Meeks III, The topology of complete minimal surfaces of finite total Gaussian curvature, Topology 22 (1983), 203-221.

[9] R. KUSNER, Conformal geometry and complete minimal surfaces, Bull. Amer. Math. Soc. 17 (1987), 291-295.

[10] - The global geometry of extremal surfaces, Ph.D thesis, Univ. California, Berkeley, 1987.

[11] S. LANG, Elliptic Functions, 2nd edition, Springer-Verlag, New York, 1987.

[12] H. B. Lawson, JR., Lectures on Minimal Subminifolds Vol. I, Publish or Perish, Inc., Berkeley, 1980.

[13] R. Osserman, Minimal surfaces in the large, Comm. Math. Helv. 35 (1961), 65-76.

[14] Global properties of minimal surfaces in $E^{3}$ and $E^{n}$, Ann. of Math. 80 (1964), 340-364.

[15] _ A Survey of Minimal Surfaces, 2nd edition, Dover Publications, New York, 1986.

[16] H. Rosenberg \& E. Toubiana, Complete minimal surfaces and minimal herissons, J. Differential Geom. 28 (1988), 115-132.

[17] R. Schoеn, Uniquness, symmetry, and embeddedness of minimal surfaces, J. Differential Geom. 18 (1983), 791-809.

[18] A. Weitsman 8 F. Xavier, Some function theoretic properties of the Gauss map for hyperbolic complete minimal surfaces, Michigan Math. J. 34 (1987), 275-283.

[19] F. XAVIER, The Gauss map of a complete non-flat minimal surface cannot omit 7 points of the sphere, Ann. of Math. 113 (1981), 211-214.

This research was partially supported by research grant DE-FG02-86ER250125 of the Applied Mathematical Science subprogram of the Office of Energy Research, U.S. Department of Energy, and Australia Research Council grant A688 30148.

Department of Mathematics

School of Mathematics Sciences

The Australian National University

GPO Box 4

Canberra ACT 2601, Australia

Received: April 7th, 1993. 